\title{
Elevated methylation levels, reduced expression levels, and frequent contractions in a clinical cohort of C9orf72 expansion carriers
}

Jazmyne L. Jackson ${ }^{1 \dagger}$, NiCole A. Finch ${ }^{1 \dagger}$, Matthew C. Baker ${ }^{1}$, Jennifer M. Kachergus², Mariely DeJesus-Hernandez ${ }^{1}$, Kimberly Pereira ${ }^{1}$, Elizabeth Christopher ${ }^{1}$, Mercedes Prudencio ${ }^{1}$, Michael G. Heckman³ ${ }^{3}$ E. Aubrey Thompson², Dennis W. Dickson ${ }^{1}$, Jaimin Shah ${ }^{4}$, Björn Oskarsson ${ }^{4}$, Leonard Petrucelli ${ }^{1}$, Rosa Rademakers ${ }^{{ }^{*}+}$ and Marka van Blitterswijk ${ }^{1 * \dagger}$ (1)

\footnotetext{
Abstract

Background: A repeat expansion in the C9orf72-SMCR8 complex subunit (C9orf72) is the most common genetic cause of two debilitating neurodegenerative diseases: amyotrophic lateral sclerosis (ALS) and frontotemporal dementia (FTD). Currently, much remains unknown about which variables may modify these diseases. We sought to investigate associations between C9orf72 promoter methylation, RNA expression levels, and repeat length, their potential effects on disease features, as well as changes over time and within families.

Methods: All samples were obtained through the ALS Center at Mayo Clinic Florida. Our primary cohort included 75 unrelated patients with an expanded C9orf72 repeat, 33 patients who did not possess this expansion, and 20 control subjects without neurodegenerative diseases. Additionally, 67 members from 17 independent C9orf72 families were selected of whom 33 harbored this expansion. Longitudinally collected samples were available for 35 C9orf72 expansion carriers. To increase our understanding of C9orf72-related diseases, we performed quantitative methylation-sensitive restriction enzyme-based assays, digital molecular barcoding, quantitative real-time PCR, and Southern blotting.

\footnotetext{
*Correspondence: Rademakers.Rosa@mayo.edu;

VanBlitterswijk.Marka@mayo.edu

${ }^{\dagger}$ Jazmyne L. Jackson and NiCole A. Finch shared first authorship.

${ }^{+}$Rosa Rademakers and Marka van Blitterswijk shared last authorship.

'Department of Neuroscience, Mayo Clinic, 4500 San Pablo Road,

Jacksonville, FL 32224, USA

Full list of author information is available at the end of the article
}

(c) The Author(s). 2020 Open Access This article is distributed under the terms of the Creative Commons Attribution 4.0 International License (http://creativecommons.org/licenses/by/4.0/), which permits unrestricted use, distribution, and reproduction in any medium, provided you give appropriate credit to the original author(s) and the source, provide a link to the Creative Commons license, and indicate if changes were made. The Creative Commons Public Domain Dedication waiver (http://creativecommons.org/publicdomain/zero/1.0/) applies to the data made available in this article, unless otherwise stated. 
(Continued from previous page)

Results: In our primary cohort, higher methylation levels were observed in patients with a C9orf72 repeat expansion than in patients without this expansion $(p=1.7 \mathrm{e}-13)$ or in control subjects $(p=3.3 \mathrm{e}-07)$. Moreover, we discovered that an increase in methylation levels was associated with a decrease in total $C 90 r f 72$ transcript levels $(p=5.5 \mathrm{e}-05)$. These findings aligned with our observation that C9orf72 expansion carriers had lower expression levels of total C9orf72 transcripts than patients lacking this expansion $(p=3.7$-07) or control subjects $(p=9.1 \mathrm{e}-05)$. We also detected an elevation of transcripts containing intron 1a (upstream of the repeat) in patients carrying a C9orf72 repeat expansion compared to (disease) controls $(p \leq 0.01)$, an indication of abortive transcripts and/or a switch in transcription start site usage. While methylation and expression levels were relatively stable over time, fluctuations were seen in repeat length. Interestingly, contractions occurred frequently in parent-offspring transmissions (> 50\%), especially in paternal transmissions. Furthermore, smaller repeat lengths were detected in currently unaffected individuals than in affected individuals $(p=8.9 \mathrm{e}-$ 04) and they were associated with an earlier age at collection $(p=0.008)$.

Conclusions: In blood from C9orf72 expansion carriers, we found elevated methylation levels, reduced expression levels, and unstable expansions that tend to contract in successive generations, arguing against anticipation.

Keywords: C9orf72, Amyotrophic lateral sclerosis, Motor neuron disease, Repeat expansion disorder, Anticipation, Hypermethylation, Expansion size, Paternal contraction,

\section{Background}

Since the discovery of a repeat expansion in the C9orf72-SMCR8 complex subunit (C9orf72) [1, 2], researchers have worked diligently to unravel the mechanisms underlying C9orf72-related diseases, including amyotrophic lateral sclerosis (ALS) and frontotemporal dementia (FTD). ALS is a neurodegenerative disorder of the upper and lower motor neurons that results in progressive muscle weakness, often leading to respiratory failure within three to five years after the onset of symptoms. FTD affects the frontal and temporal lobes, causing dementia characterized by changes in personality, behavior, and/or language deficits. There are three proposed mechanisms by which an expanded C9orf72 repeat might act: reduced gene expression, accumulation of RNA foci, and/or aggregation of dipeptide repeat proteins [1, 3-7].

Although methylation of the C9orf72 promoter, expression levels of $C 90$ rf 72 transcripts, and the length of the hexanucleotide expansion have been studied in blood [7-18], they have not been evaluated together in a single comprehensive large-scale study. Hence, we set out to perform a thorough characterization of our clinical cohort, which enabled us to examine correlations between these variables and to determine whether they are associated with features of C9orf72-linked diseases. Moreover, because we collected specimens longitudinally and from multiple family members, we were able to investigate changes over time and within families. Our extensive assessment of this cohort may help to improve our understanding of these complicated neurodegenerative disorders.

\section{Methods}

\section{Participants}

All biological specimens were collected at our ALS Center at Mayo Clinic Florida between 2008 and 2018. Our primary cohort consisted of 75 unrelated patients harboring a C9orf72 repeat expansion (> 93\% ALS), 33 ALS patients who did not carry the expansion, and 20 control subjects who had neither been diagnosed with ALS nor harbored a repeat expansion $(n=128$; Table 1$)$. We added 33 expansion carriers and 34 subjects without this expansion from C9orf72 families to create our overall cohort $(n=195)$, containing 108 expansion carriers (Additional file 1: Table S1). Our overall cohort included 17 families with at least two members $(n=87)$. In addition, multiple time-points were collected for 35 C9orf72 expansion carriers with up to seven time-points.

\section{C9orf72 methylation}

For our overall cohort $(n=195)$, DNA was extracted from blood using the Gentra Puregene Kit (Qiagen). Additionally, for a subset of individuals $(n=14)$, DNA was extracted from the frontal cortex and cerebellum with standard phenol/chloroform procedures. To determine methylation levels of the C9orf72 promoter, a quantitative assay was performed as described previously [11]. In brief, $100 \mathrm{ng}$ of genomic DNA was digested for $16 \mathrm{~h}$ with 2 units of both HhaI and HaeIII (New England BioLabs; experimental condition) or with 2 units of HaeIII (control condition), followed by heat inactivation. A quantitative real-time PCR was done on a Quantstudio 7 (Applied Biosystems) and methylation levels were estimated. 


\section{C9orf72 expression}

Digital molecular barcoding was carried out on the nCounter system (NanoString Technologies) [19]. Briefly, for our expression cohort $(n=87)$, RNA was extracted from blood using the PAXgene Blood RNA Kit (PreAnalytiX) and the quality was determined on a 2100 Bioanalyzer (Agilent Technologies). Subsequently, 250 ng was used to assess total C9orf72 transcripts, variant 1 transcripts (NM_145005.6), variant 2 transcripts (NM_ 018325.4), and intron containing transcripts (intron 1a [upstream of the repeat] and intron $1 \mathrm{~b}$ [downstream of the repeat]). Hypoxanthine phosphoribosyltransferase 1 (HPRT1) and tyrosine 3-monooxygenase/tryptophan 5monooxygenase activation protein zeta (YWHAZ) were used as endogenous controls.

Additionally, for a specific family (PED1) and matched controls, gene expression assays (TaqMan) were performed [19]. Approximately $200 \mathrm{ng}$ of template RNA was used to generate complimentary DNA (cDNA) with the SuperScript III Kit (Invitrogen). Quantitative realtime PCR was then undertaken on a Quantstudio 7 for total C9orf72 transcripts (Hs00376619_m1), C9orf72 variant 1 (custom assay), and C9orf72 variant 2 (custom assay), using HPRT1 (Hs02800695_m1) and YWHAZ (Hs00852925_sH) as endogenous controls.

\section{C9orf72 repeat length}

High-quality DNA from blood was available for C9orf 72 expansion carriers $(n=97)$ from our overall cohort; DNA from the frontal cortex and cerebellum was obtained for a subset of individuals $(n=14)$. Southern blotting was performed as described elsewhere [14]. In short, approximately $10 \mu \mathrm{g}$ of genomic DNA was digested with restriction enzyme XbaI (Promega Corporation), electrophoresed, transferred to a positively charged nylon membrane (Roche), and crosslinked via ultraviolet irradiation. After pre-hybridization, the membrane was hybridized with a digoxigenin (DIG)-labelled probe (Roche). To reduce the background signal, stringency washes and subsequent blocking were performed. An antiDIG antibody (1:10,000; Roche) was utilized to allow visualization on autoradiography film. Expansion sizes were estimated using AlphaEase FC (Alpha Innotech).

\section{Statistical analysis}

The three primary measures of this study (methylation levels, expression levels, and repeat lengths) were compared between groups using a Kruskal-Wallis rank sum test, a Wilcoxon rank sum test, or a paired Wilcoxon signed-rank test, as appropriate for a given comparison. Correlations between continuous variables were assessed using a Spearman's test of correlation; Spearman's correlation coefficient $r$ was estimated. A linear regression model was used with repeat length as outcome to determine the presence of an association with the disease status (affected versus unaffected), when adjusting for age at collection. Cox proportional hazards regression models were used to examine associations between the three primary measures and survival after onset, where measures were dichotomized using the median, models were adjusted for age at onset, and censoring occurred at the date of last follow-up. Changes in the three primary measures over time were examined utilizing mixed effects linear regression models, including a fixed effect for time and a random effect for each individual. When more than one measurement was available for a subject and/or time-point, the mean of those measurements was used. To adjust for multiple testing, a Bonferroni correction was utilized, separately for each group of similar statistical tests. All statistical tests were two-sided and were performed using R (v3.5.3).

\section{Results}

\section{Hypermethylation of C9orf72 promoter}

Methylation levels of the C9orf 72 promoter were determined in blood for our primary cohort (Table 1). A significant difference in methylation levels was observed between groups ( $p=4.5 \mathrm{e}-15$; Table 2). C9orf72 expansion

Table 1 Characteristics of primary cohort

\begin{tabular}{llll}
\hline Variable & C9Plus $(n=75)$ & C9Minus $(n=33)$ & Control $(n=20)$ \\
\hline Sex, No. (\% female) & $41(54.67)$ & $15(45.45)$ & $10(50.00)$ \\
Site of Onset, No. (\% bulbar) & $15(20.00)$ & $6(18.18)$ & NA \\
Age at Collection, median (IQR), y & $60.87(55.28-65.94)$ & $61.25(56.58-65.05)$ & $60.05(52.05-65.26)$ \\
Age at Onset, median (IQR), y & $59.25(52.94-64.08)$ & $60.08(55.08-63.42)$ & NA \\
Survival after Onset ${ }^{a}$, median (IQR), y & $1.98(1.54-3.10)$ & $1.99(1.12-3.19)$ & NA \\
Methylation, median (IQR), \% & $4.05(1.10-17.95)$ & $0.24(0.15-0.33)$ & $0.46(0.14-0.75)$ \\
Expression, median (IQR), \% & $73.32(65.96-86.20)$ & $110.70(93.68-132.88)$ & $100.00(87.48-111.27)$ \\
Repeat Length, median (IQR), kb & $20.05(15.58-26.27)$ & $\mathrm{NA}$ & $\mathrm{NA}$ \\
\hline
\end{tabular}

C9Plus patients with a C9orf72 repeat expansion, C9Minus patients without this expansion, Control control subjects without a neurodegenerative disease, IQR interquartile range, $N A$ not applicable

${ }^{a}$ In total, 23 of our 75 affected C9orf72 expansion carriers are currently alive (31\%). Of the 33 patients without this expansion, 20 are alive (61\%) 
carriers had median methylation levels of $4.1 \%$, which was significantly higher than non-expansion carriers $(0.2 \%$, $p=1.7 \mathrm{e}-13)$ and controls $(0.5 \%, p=3.3 \mathrm{e}-07$; Fig. 1a). With increasing methylation levels, there was a decrease in expression levels of total C9orf72 transcripts $(r:-0.42, p=$ 5.5e-05), variant 1 transcripts $(r:-0.35, p=9.5 \mathrm{e}-04)$, variant 2 transcripts $(r:-0.35, p=8.7 \mathrm{e}-04)$, and intron $1 \mathrm{~b}$ containing transcripts $(r:-0.30, p=0.005)$. Within the subset of individuals with a C9orf72 repeat expansion, we found hypermethylation in $36.0 \%$, when using a threshold of $10.0 \%$ [11]. Similar findings were obtained in our overall cohort that included additional members from $C 9$ orf 72 families (Additional file 1: Table S1). We did not detect a significant difference between $C 90 r f 72$ expansion carriers with or without symptoms $(p=0.17)$.

To determine whether methylation levels of the C9orf72 promoter were stable over time, we subsequently assayed longitudinally collected blood specimens. Although some variability in promoter methylation over time was observed, subjects with low methylation levels remained low, while subjects with relatively high methylation levels remained high ( $p=$ 0.56; Fig. 1b). These findings were further substantiated by our assessment of $C 9$ orf 72 expansion carriers who had died and for whom brain tissue was available $(n=$ 14): methylation levels in blood correlated with those in the frontal cortex $(r: 0.56, p=0.04)$ or cerebellum $(r$ : 0.81, $p=3.8 \mathrm{e}-04$; Fig. 1c, Additional file 1: Figure S1a-b).

\section{Reduced expression of C9orf72 transcripts}

RNA expression levels of C9orf72 transcripts in blood were evaluated in our expression cohort (Additional file 1: Table S2). For total C9orf72 transcripts, a significant difference was detected between groups $(p=2.1 \mathrm{e}-07$; Table 2, Fig. 2a). Specifically, median expression levels were lower in C9orf72 expansion carriers (73.3\%) compared to non-expansion carriers $(110.7 \%, p=3.7 \mathrm{e}-07)$ and controls (100.0\%, $p=9.1 \mathrm{e}-05)$. A similar pattern was seen for variant 1 transcripts, variant 2 transcripts, and intron $1 \mathrm{~b}$ containing transcripts $(p \leq 0.003)$. Interestingly, intron 1a containing transcripts $(p=8.4 \mathrm{e}-04)$ demonstrated elevated levels in C9orf72 expansion carriers when comparing them to (disease) controls ( $p \leq 0.01$; Table 2). For total C9orf72 transcripts, no significant difference was observed between affected and unaffected expansion carriers $(p=0.63)$.

Assessment of longitudinal changes in RNA expression levels uncovered that most C9orf72 expansion carriers had low expression levels in blood that were stable over time $(p=0.47$; Fig. $2 \mathrm{~b})$. One individual, however, was consistently identified as an outlier with relatively high total C9orf72 expression levels (>200\%). This individual was an otherwise typical ALS patient with an age at onset of 56 years and a survival of about 2 years after the onset of symptoms. The Southern blot of this person displayed two bands, including a weak band of $7.9 \mathrm{~kb}(\sim$ 900 repeats $)$ and a smaller, sharper band of $3.4 \mathrm{~kb}(\sim 200$ repeats; Additional file 1: Figure S2a).

\section{Instability of C9orf72 repeat}

We estimated the repeat length of 64 C9orf72 expansion carriers in our primary cohort for whom sufficient highquality DNA was available from blood. In these expansion carriers, the median repeat length was $20.1 \mathrm{~kb}(\sim$ 3000 repeats). Additionally, we included 33 extra expansion carriers from our overall cohort, resulting in a median repeat length of $18.2 \mathrm{~kb} \quad(\sim 2700$ repeats $)$. Importantly, when comparing C9orf 72 expansion carriers who were affected $(n=73)$ to expansion carriers who were not (yet) affected $(n=24)$, a significant difference was observed: affected expansion carriers had a repeat length of $20.5 \mathrm{~kb}(\sim 3000$ repeats $)$ versus $13.7 \mathrm{~kb}(\sim$ 1900 repeats) in unaffected expansion carriers $(p=8.9 \mathrm{e}-$ 04; Fig. 3a). To determine whether this association could

Table 2 Methylation and expression levels of C9orf72

\begin{tabular}{|c|c|c|c|c|c|c|c|}
\hline \multirow[b]{2}{*}{ Variable } & \multicolumn{3}{|l|}{ Groups } & \multicolumn{4}{|l|}{ P-Values } \\
\hline & C9Plus & C9Minus & Control & Groups $^{a}$ & $\begin{array}{l}\text { C9Plus vs } \\
\text { c9Minus }^{\text {b }}\end{array}$ & $\begin{array}{l}\text { C9Plus vs } \\
\text { Control }^{\text {b }}\end{array}$ & $\begin{array}{l}\text { C9Minus vs } \\
\text { Control }^{\text {C }}\end{array}$ \\
\hline Methylation, median (IQR), \% & $4.05(1.10-17.95)$ & $0.24(0.15-0.33)$ & $0.46(0.14-0.75)$ & $4.45 e-15$ & $1.70 \mathrm{e}-13$ & $3.33 e-07$ & 0.17 \\
\hline Total, median (IQR), \% & $73.32(65.96-86.20)$ & $110.70(93.68-132.88)$ & $100.00(87.48-111.27)$ & $2.06 \mathrm{e}-07$ & $3.67 e-07$ & $9.08 \mathrm{e}-05$ & 0.13 \\
\hline Variant 1, median (IQR), \% & $55.66(48.14-72.82)$ & $80.10(70.31-94.64)$ & $100.00(74.87-110.67)$ & 1.07e-05 & $2.83 e-04$ & $3.65 e-05$ & 0.06 \\
\hline Variant 2, median (IQR), \% & $61.97(55.23-73.67)$ & $104.75(85.86-139.38)$ & $100.00(90.04-120.12)$ & $9.66 \mathrm{e}-08$ & $3.43 e-07$ & $1.10 \mathrm{e}-05$ & 0.76 \\
\hline Intron 1a, median (IQR), \% & $411.20(244.09-637.62)$ & $263.06(103.15-398.10)$ & 100.00 (37.09-290.55) & $8.40 \mathrm{e}-04$ & 0.01 & $4.03 e-04$ & 0.13 \\
\hline Intron $1 \mathrm{~b}$, median (IQR), \% & $61.18(54.37-90.30)$ & 98.61 (75.58-109.73) & $100.00(67.11-130.03)$ & 0.003 & 0.002 & 0.01 & 0.48 \\
\hline
\end{tabular}

C9Plus patients with a C9orf72 repeat expansion, C9Minus patients without this expansion, Control control subjects without a neurodegenerative disease, IQR interquartile range

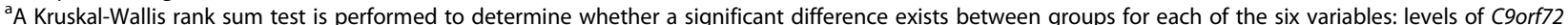
promoter methylation (Methylation), total C9orf72 transcripts (Total), variant 1 transcripts (Variant 1), variant 2 transcripts (Variant 2), intron 1a containing transcripts (Intron 1a), and intron 1b containing transcripts (Intron $1 \mathrm{~b} ; p<0.008$ is considered significant after Bonferroni correction)

${ }^{\mathrm{b}} \mathrm{A}$ Wilcoxon rank sum test is used when the Kruskal-Wallis test is significant for each of the three pairwise comparisons: C9Plus vs C9Minus, C9Plus vs Control, and C9Minus vs Control ( $p<0.017$ is considered significant after Bonferroni correction) 

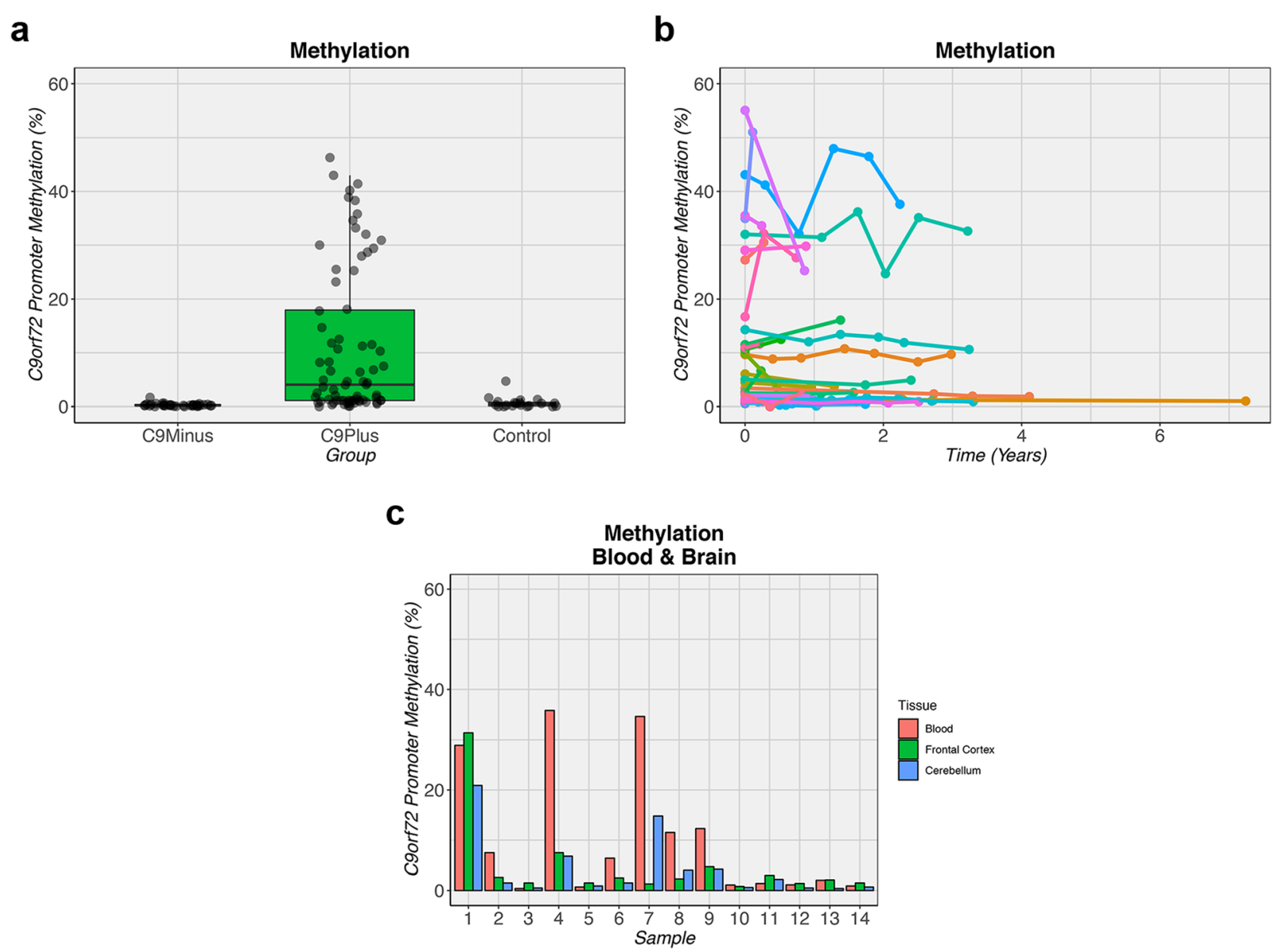

Fig. 1 Methylation of C9orf72 promoter. a Increased methylation levels of the C9orf72 promoter are observed when comparing patients with a C9orf72 repeat expansion (C9Plus) to patients without this expansion (C9Minus) or to control subjects (Control). The median is represented by a solid black line, and each box spans the interquartile range (IQR; 25th percentile to 75th percentile). b Methylation levels appear to be fairly stable over time: subjects with high methylation levels remain high, while those with low levels remain low. For each individual, longitudinal measurements are connected by a solid colored line. $\mathbf{c}$ In blood, frontal cortex, and cerebellum, a similar methylation pattern is seen. For each individual, three bars are displayed that correspond to methylation levels in a specific tissue type

have been driven by a difference in age at collection, we performed a linear regression analysis with repeat length as outcome. When including the disease status (affected versus unaffected) as a single variable, a significant association was detected $(p=0.001)$. This association remained significant $(p=0.01)$, after adding age at collection to our model $(p=0.80)$. We did not detect a significant correlation between the repeat length and methylation or expression levels $(p \geq 0.31)$.

Next, we examined blood specimens collected at multiple time-points. In individual cases, the length did vary; however, in general, the repeat length did not change over time ( $p=0.50$; Fig. 3b). One individual, for instance, exhibited a minor increase of $0.5 \mathrm{~kb}(\sim 100$ repeats) over a period of more than 7 years (Additional file 1: Figure S2b). For a subset of $C 9$ orf72 expansion carriers $(n=14)$, repeat lengths were also estimated in autopsy tissues, which did not uncover a correlation between lengths in blood and brain ( $p \geq 0.44$; Fig. 3c, Additional file 1: Figure S1c-d).

\section{Detection of clinical associations}

We restricted our analysis to unrelated patients harboring an expanded C9orf72 repeat to evaluate associations with clinical features, including age at onset, age at collection, site of onset, sex, and survival after onset. After adjustment for multiple testing, no significant associations were detected with methylation levels, expression levels, or repeat lengths (data not shown). When we added affected $(n=9)$ and unaffected $(n=24)$ family members carrying the expansion, however, we did detect a significant association between the length of the repeat and age at collection $(r: 0.27, p=0.008)$, indicating that smaller expansions are present in younger individuals. 

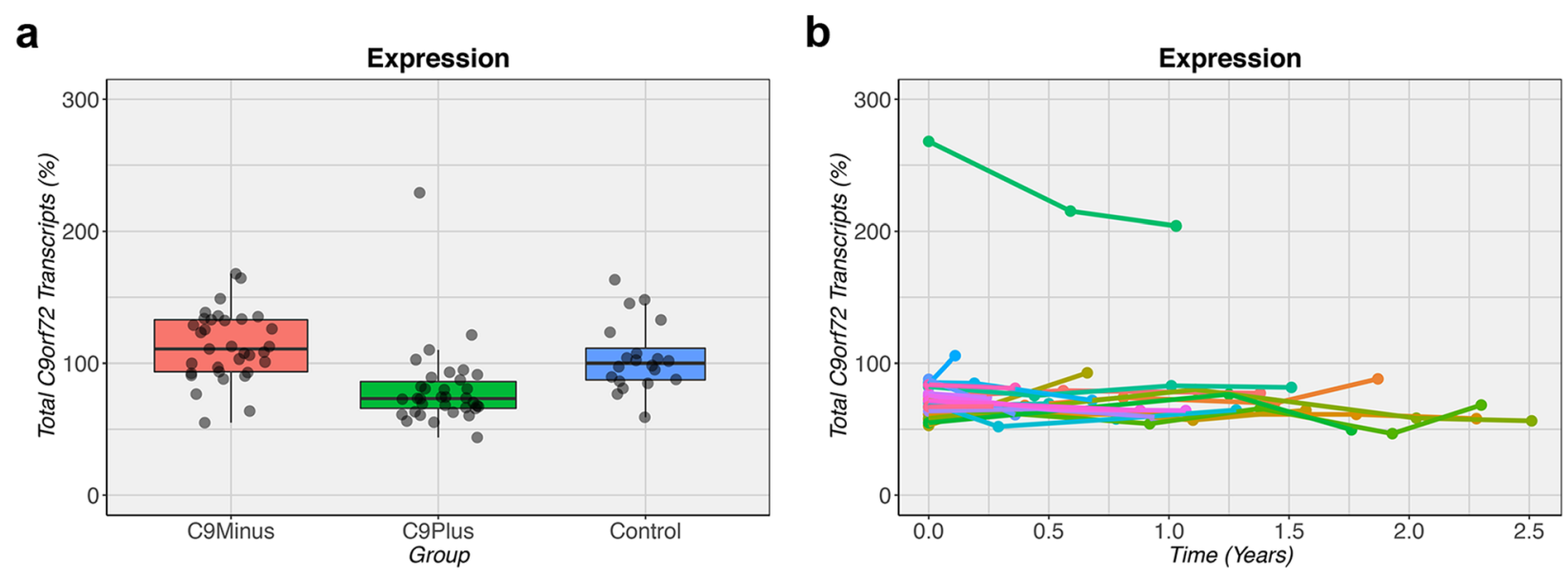

Fig. 2 Expression of C9orf72 transcripts. a In patients with an expanded C9orf72 repeat (C9Plus), the expression levels of total C9orf72 transcripts are lower than in patients without this expansion (C9Minus) or in control subjects (Control). The median is represented by a solid black line, and each box spans the interquartile range (IQR; 25th percentile to 75th percentile). b Over time, the levels of total C9orf72 transcripts remain relatively stable. One outlier is detected with levels that are consistently higher than in other C9orf72 expansion carriers. This outlier has an expansion of $7.9 \mathrm{~kb}$ ( $\sim 900$ repeats) in addition to an expansion of $3.4 \mathrm{~kb}(\sim 200$ repeats). For each individual, longitudinal measurements are connected by a solid colored line

\section{Description of families}

To examine changes within families, we then compared methylation levels, expression levels, and repeat lengths in parent-offspring transmissions. In 14 out of 17 transmissions (82.4\%) from 10 unrelated families, hypermethylation was either present or absent (Fig. 4, Fig. 5). Of the remaining transmissions, two (11.8\%) demonstrated a change in status from lowly methylated to hypermethylated and one (5.9\%) from hypermethylated to lowly methylated. In seven out of 10 transmissions where RNA was available (70.0\%), expression levels appeared to be relatively stable. In the remaining three transmissions (30.0\%), we detected an elevation in transcript levels of children with an expansion size of less than $5 \mathrm{~kb}$ (Fig. 5a, Fig. 5c). Interestingly, contracted expansions were commonly encountered and present in 10 out of 17 parent-to-child transmissions (58.8\%; Fig. 4, Fig. 5, and Additional file 1: Figure S2c); all these transmissions exhibited a difference of more than $5 \mathrm{~kb}$ between generations and the majority were paternal (90.0\%). An increase in expansion size was less common and seen in two maternal transmissions (11.8\%), while a stable expansion was observed in five maternal transmissions (29.4\%). When comparing expansion sizes between parents and children from these 17 parent-offspring pairs, the expansion size was significantly longer in parents than in children $(p=0.01)$. Similar findings were obtained when restricting the analysis to one parent-to-child transmission per parent $(n=12)$.

One of the families with a contracted expansion, PED1, demonstrated changes between the proband (II1), his siblings (II-3 and II-4), and his children (III-1 and
III-2; Fig. 3d). The proband had been diagnosed with ALS in his late fifties and died after a disease duration of approximately 4 years. One of his siblings also developed ALS in his fifties, while another sibling was currently unaffected, just like his children who were relatively young (in their thirties). When examining methylation levels of the C9orf72 promoter in this family, low levels were observed in all family members. Expression levels, however, differed: the proband had a total C9orf72 transcript level of $64.5 \%$, his siblings of 49.5 and $51.4 \%$, and his children of 126.2 and $182.2 \%$, when comparing them to controls matched based on sex and age (100.0\%). Examination of the repeat length revealed that the proband harbored an expansion of $12.3 \mathrm{~kb}$ ( 1700 repeats), his affected sibling of $8.4 \mathrm{~kb}(\sim 1000$ repeats), and his unaffected sibling of $10.7 \mathrm{~kb}(\sim 1400$ repeats $)$. His children were found to carry a contracted expansion with repeat lengths of 2.8 $\mathrm{kb}(\sim 100$ repeats $)$ and 5.9/3.3 kb ( 600/200 repeats), respectively (Additional file 1: Figure S2d).

\section{Discussion}

Our characterization of C9orf72 expansion carriers in blood showed that $36 \%$ demonstrate hypermethylation of the C9orf72 promoter. Additionally, we detected reduced expression levels of C9orf72 transcripts and, when comparing expansion sizes between affected and unaffected individuals, we found a difference of roughly 7 $\mathrm{kb}$ (> 1000 repeats). Investigation of longitudinal changes uncovered relatively stable methylation and expression levels; however, fluctuations were seen in the size of the expansion. Similarly, in parent-offspring transmissions, little variation was observed in methylation and 


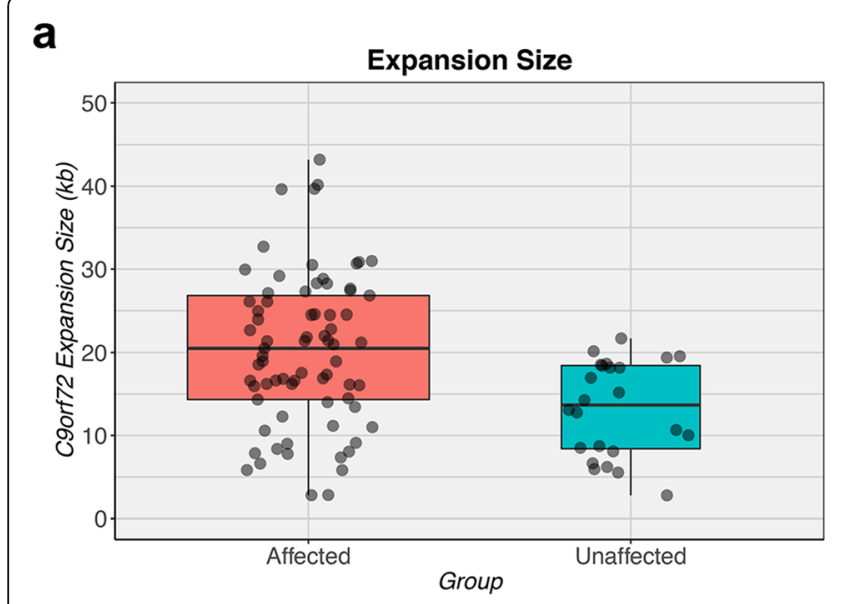

b
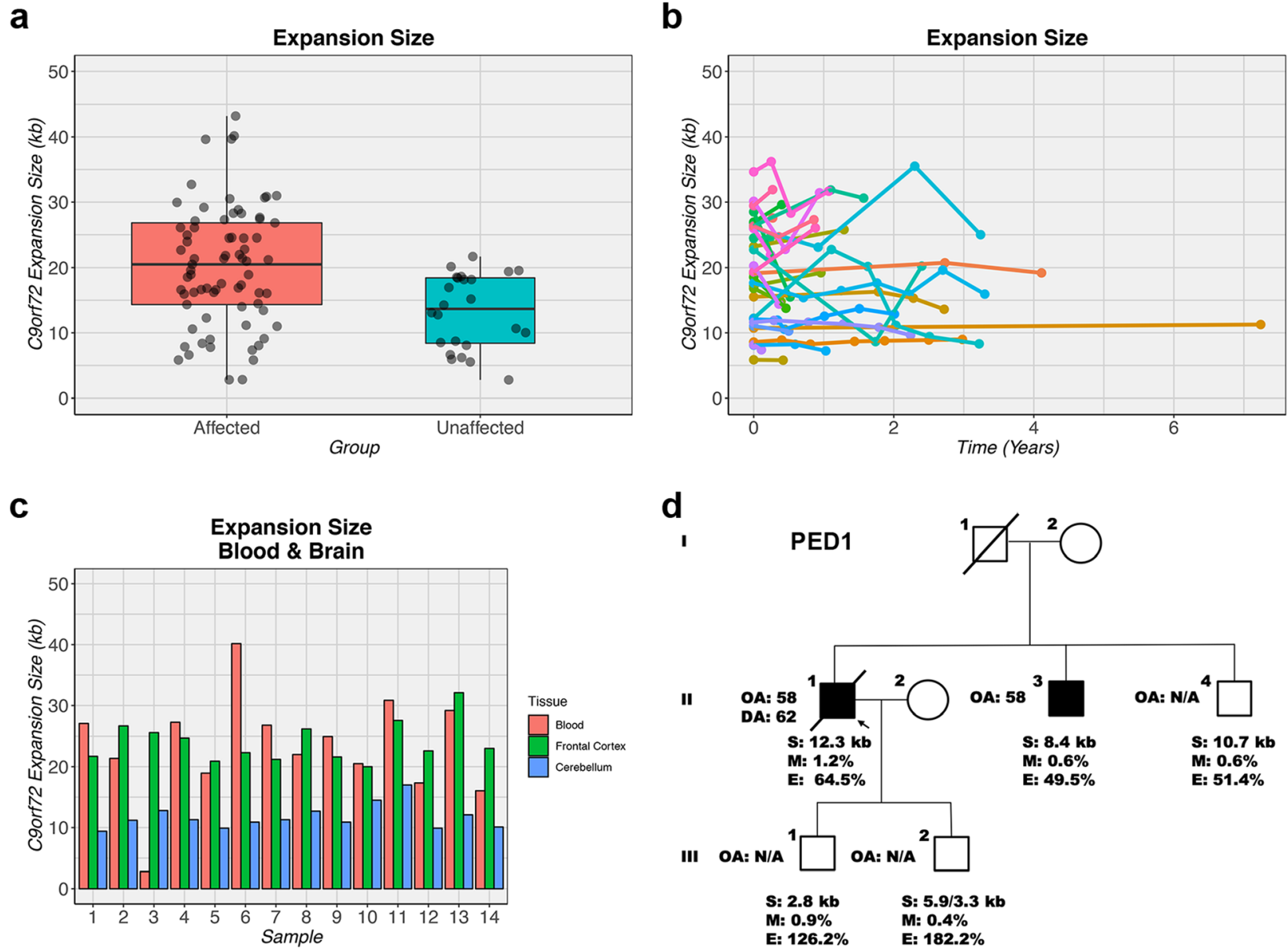

d

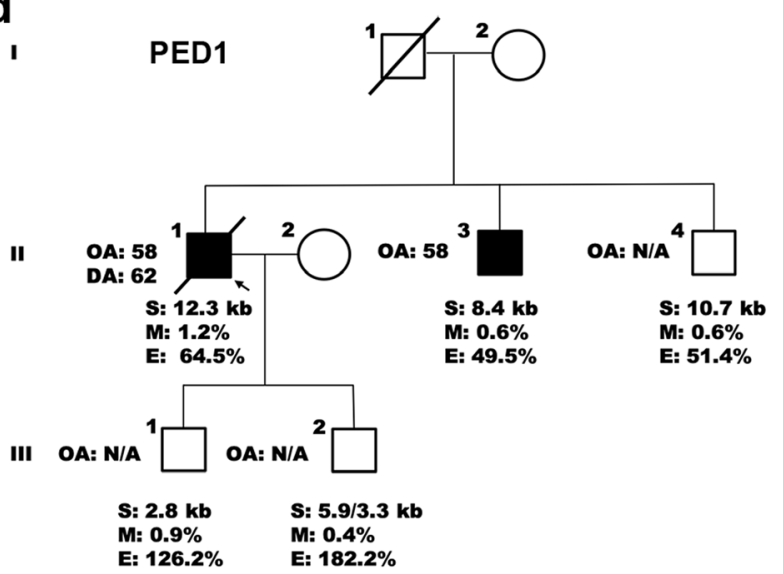

Fig. 3 Expansion size of C9orf72 repeat. a When comparing C9orf72 expansion carriers with symptoms (affected) to those presently without symptoms (unaffected), a longer expansion is observed in affected subjects. The median is represented by a solid black line, and each box spans the interquartile range (IQR; 25 th percentile to 75 th percentile). b Fluctuations are detected in the expansion size of subjects with an expanded C9orf72 repeat; no clear pattern emerges. For each individual, longitudinal measurements are connected by a solid colored line. c There is no correlation between expansion sizes in blood, frontal cortex, or cerebellum. For each individual, three bars are displayed that correspond to expansion sizes in a specific tissue type. $\mathbf{d}$ One pedigree is shown (PED1) with the proband (II-1; arrow), his siblings (II-3 and II-4), and his children (III-1 and III-2). Affected individuals are denoted by a solid black square. Information is provided regarding the onset age (OA), death age (DA), expansion size (S), methylation level (M), and expression level (E), when available (N/A)

expression, whereas instability was detected in the length of the repeat, which contracted in the majority of cases.

The hypermethylation frequency we detected is in agreement with previous studies that investigated blood from ALS patients $[8,11]$. We also discovered that the methylation status was fairly stable over time and within families. These findings were substantiated by our detection of correlations between methylation levels in blood and brain, confirming other reports $[8,11]$. The C9orf 72 expression pattern we found in blood was similar to that described in the frontal cortex as well [19]: in both regions, we demonstrated a decrease in most C9orf72 transcripts. Additionally, we noticed an upregulation of transcripts containing intron 1a, an intronic area located just before the repeat. While transcripts containing intron $1 \mathrm{~b}$ (after the repeat) were reduced, this seems to indicate that the expanded repeat might trigger the production of abortive transcripts $[19,20]$ and/or a switch in transcription start site usage [21]. Intriguingly, in individuals with small repeat sizes $(<5 \mathrm{~kb})$, we detected relatively high $C 9$ orf 72 expression levels, including an outlier with expression levels above $200 \%$. These findings are in accordance with other descriptions of subjects with intermediate or small expansions who exhibit normal to elevated expression levels [8, 22-24]. In general, though, we did not detect an association between the length of the C9orf 72 repeat expansion and methylation or expression levels, which contradicts other reports [11].

Importantly, our examination of expansion sizes in blood revealed a pattern different from that seen in brain tissue [14]. Individual cases where discrepancies were 
a

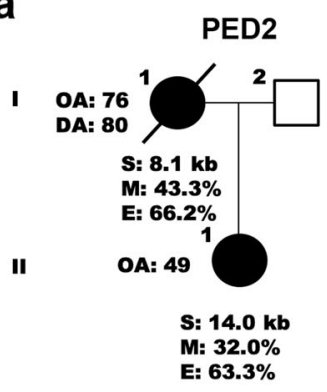

d

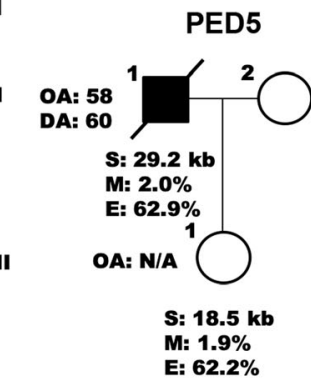

g

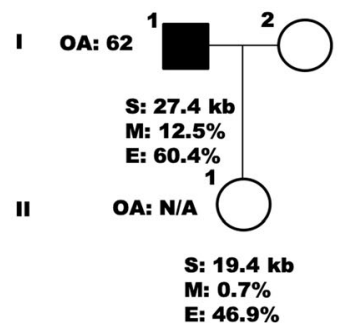

b

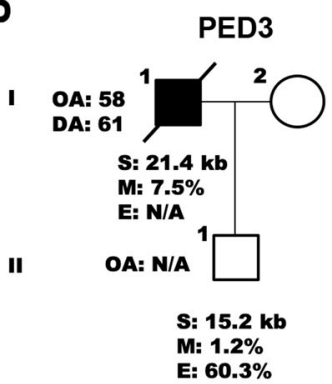

e

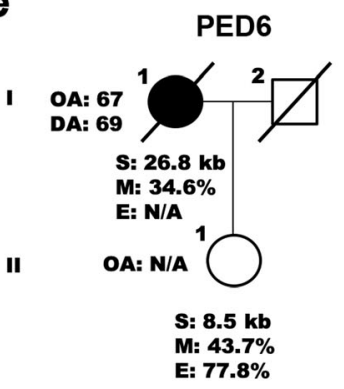

h

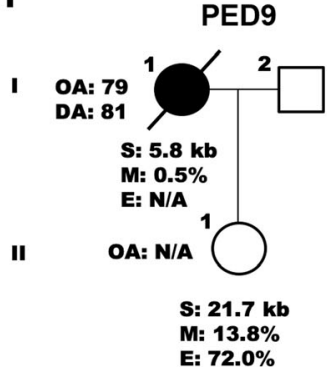

C

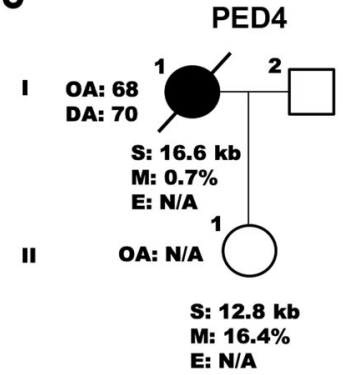

f
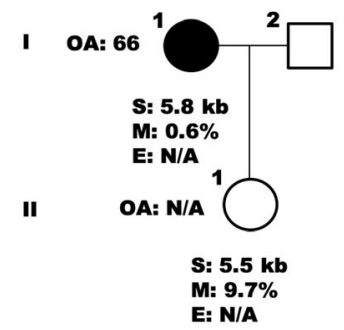

i

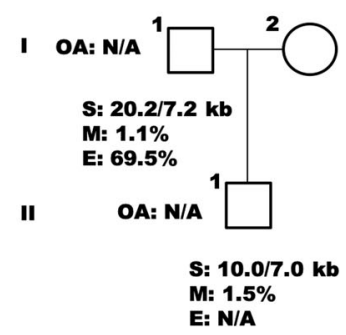

Fig. 4 Parent-offspring transmissions I. a - i This figure contains nine parent-offspring transmissions (PED2 to PED10). The last two transmissions (PED9 and PED10) occurred in a different branch of the same family. Affected individuals are denoted by a solid black circle or square. Information is provided regarding the onset age $(\mathrm{OA})$, death age (DA), expansion size (S), methylation level $(\mathrm{M})$, and expression level (E), when available (N/A)

noted between sizes in blood and brain have already been described by us and others [14, 16, 22, 25]. Together, these findings suggest that repeat lengths measured in blood, an unaffected region, might not mirror lengths in the central nervous system, possibly due to somatic mosaicism. Despite the fact that blood can be useful in establishing the presence or absence of $C 9$ orf 72 repeat expansions, our findings indicate that one should be careful when interpreting size estimates based on blood measurements.

In several repeat expansion disorders, such as myotonic dystrophy [26], anticipation has been described where a more severe phenotype is thought to arise from an increase in the length of the repeat in successive generations. One study suggested that the age at onset in patients with an expanded C9orf 72 repeat decreases from one generation to the next [27]. These findings align with another report that detected an increase in expansion size when transmitting the repeat from parent to child [18]. Although these studies could indeed point to anticipation, our work suggests that this may not be the case. In successive generations, we actually noticed that the repeat expansion had a tendency to contract, which was also reported by others [12]. One wonders, therefore, whether the earlier age at onset reported in the former study might be a reflection of selection bias, recall bias, and/or diagnostic bias. Furthermore, it seems plausible that, when a disease runs in a given family, this could raise awareness in family members who may seek medical attention sooner and who may receive a diagnosis at an earlier age. Additionally, the increase in expansion size observed in the latter study depends on one parent-to-child transmission. The relatively high frequency of contractions we detected in a larger number of parent-offspring transmissions, mainly in paternal transmissions, is similar to that described in Friedreich's 
a

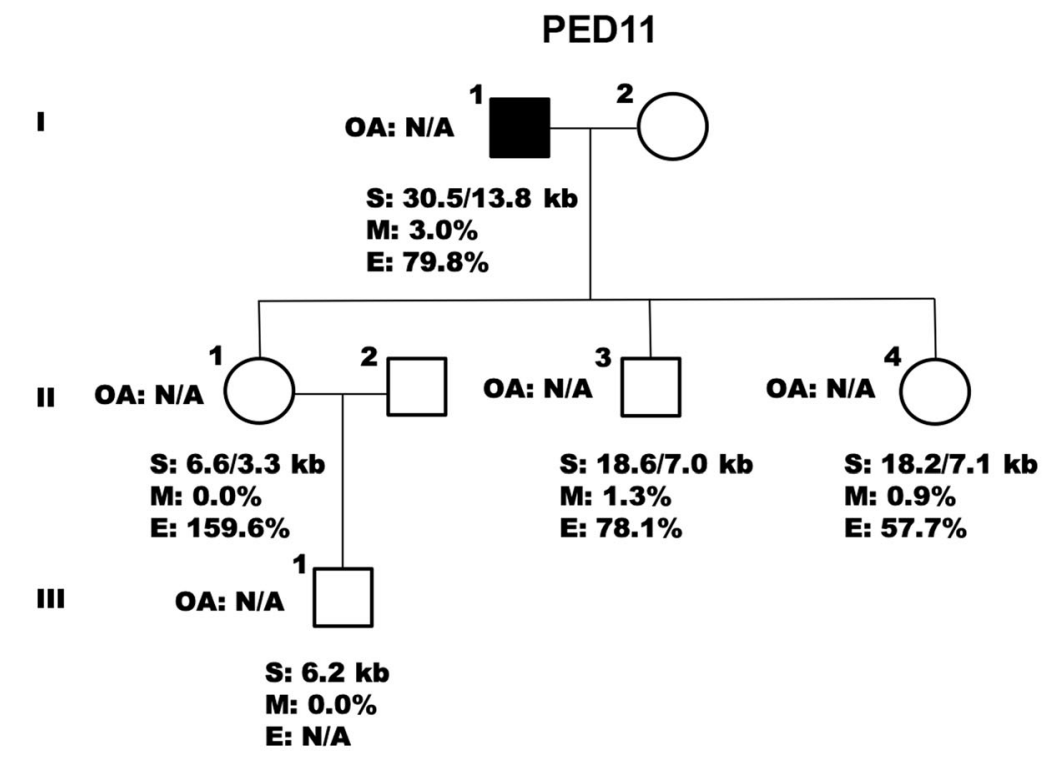

b

PED12

I

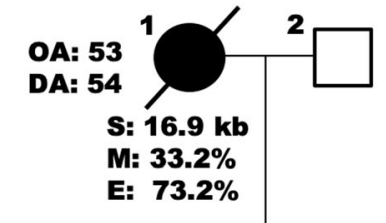

II

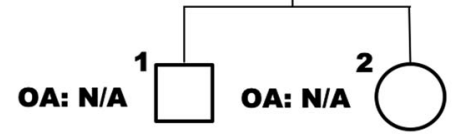

S: $13.1 \mathrm{~kb}$

M: $\mathbf{4 1 . 5 \%}$

E: $\mathbf{7 2 . 3 \%}$
S: $18.2 \mathrm{~kb}$

M: $\mathbf{3 5 . 8} \%$
C

I

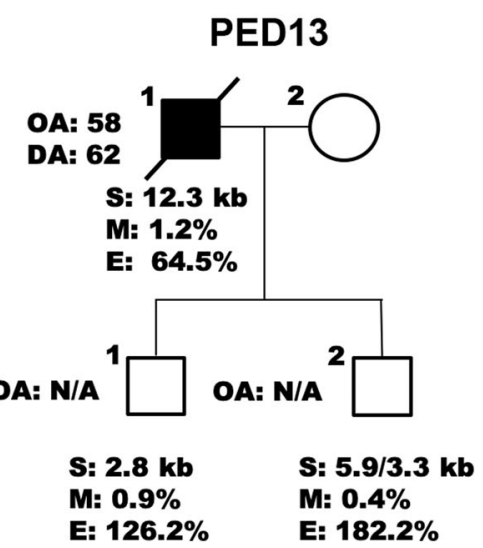

Fig. 5 Parent-offspring transmissions II. a - c Three pedigrees (PED11 to PED13) are displayed with additional parent-offspring transmissions (eight in total). The first pedigree (PED11) represents another branch from a family with multiple branches (PED9 and PED10; Fig. 4h-i). The last pedigree (PED13) is a simplified version of a pedigree shown elsewhere (PED1; Fig. 3d). Affected individuals are denoted by a solid black circle or square. Information is provided regarding the onset age (OA), death age (DA), expansion size (S), methylation level (M), and expression level (E), when available (N/A)

ataxia where paternally inherited expansions generally decrease in size [28]. Based on our observation that testes can contain a small contracted expansion in addition to a long expansion [14], we hypothesize that the presence of such a contraction in male germ cells might explain why contractions are primarily seen in paternal transmissions. Notably, it is possible that expansions are relatively stable in germ cells, whereas they demonstrate instability in somatic cells. The biological relevance of contractions remains unclear and should be investigated in future studies.

Our discovery of contracted expansions is supported by our detection of significantly smaller expansions in presently unaffected individuals than in affected individuals. Moreover, it also agrees with the association we found between the length of the expansion and age at collection in our extended cohort of C9orf72 expansion carriers. Basically, these findings suggest that contractions occur frequently and that, as a consequence, the repeat length is smaller in young expansion carriers who are currently unaffected. It should be noted, though, that the association with disease status (affected versus unaffected) seemed more robust than that with age at collection. Over time, the length of the expansion may fluctuate, exhibiting both contractions and expansions. This instability could result in profound differences in 
repeat length, and hence, the length in blood at a given point in time may be a poor reflection of the original length. As such, this could explain conflicting results reported in the literature, depending on the time-points or tissues analyzed as well as the number of unaffected individuals studied [11, 12, 14, 17].

\section{Limitations}

Although we performed a thorough characterization of our clinical cohort of subjects harboring an expanded C9orf72 repeat, we realize that the methods we employed and the specimens we collected have limitations. The methylation-sensitive restriction enzymebased assay we utilized, for instance, depends on the methylation status of a single CpG. Despite the fact that this assay has been validated using bisulfite sequencing and has been shown to provide a good estimate of the entire promoter area [11], we cannot rule out the possibility that other CpG sites are methylated. Because RNA was not available for all subjects included in our study, we could only obtain C9orf72 transcript levels from a subset of individuals. It should also be noted that C9orf72 transcript variant 3 was not assessed, since it cannot be detected reliably using digital molecular barcoding [19]. Additionally, we acknowledge that Southern blots are challenging and that the presence of a smear, frequently detected in blood, can hamper its accuracy. To improve our estimates, we did measure our samples multiple times $(2.4 \mathrm{x}$, on average) and the degree of variability in repeat lengths was relatively low (median subject-specific standard deviation: $1.9 \mathrm{~kb}[\sim 300$ repeats]). Because we focused on blood, we cannot exclude the possibility that our findings would have been different if we had evaluated an affected region. Given the limited availability of brain tissue from multiple generations, for example, it is currently unclear whether the contractions we detected in blood can be observed in brain tissue. It should also be stressed that due to the relatively short survival after onset of ALS patients, it is difficult to collect longitudinal samples from affected individuals over an extended period of time, which might have influenced our ability to detect significant changes over time. Lastly, our study mainly included ALS patients, and therefore, additional studies will have to be performed to determine whether similar findings can be obtained in FTD patients.

\section{Conclusions}

In this comprehensive blood-based study, we evaluated the methylation status, expression level, and repeat length in a clinical cohort of C9orf72 expansion carriers. We detected hypermethylation of the C9orf72 promoter and reduced expression levels of C9orf 72 transcripts, which were both stable over time and within families.
The length of the repeat, on the other hand, demonstrated more variability and was not comparable to that detected in other regions; in parent-to-child transmissions, contractions were more commonly encountered than expansions, arguing against anticipation in C9orf72-linked diseases.

\section{Supplementary information}

Supplementary information accompanies this paper at https://doi.org/10. 1186/s13024-020-0359-8.

Additional file 1 Table S1. Characteristics of overall cohort; Table S2 Characteristics of expression cohort; Figure S1. Comparison blood and brain; Figure S2. Southern blot examples.

\section{Abbreviations}

ALS: Amyotrophic lateral sclerosis; C9orf72: C9orf72-SMCR8 complex subunit; DIG: Digoxigenin; FTD: Frontotemporal dementia; HPRT1: Hypoxanthine phosphoribosyltransferase 1; YWHAZ: Tyrosine 3-monooxygenase/tryptophan 5-monooxygenase activation protein zeta

\section{Authors' contributions}

JJ and NAF: acquired data, analyzed or interpreted data, drafted the manuscript for intellectual content, and revised the manuscript for intellectual content. MCB, JMK, MDJ, KP, EC, and MP: acquired data, analyzed or interpreted data, and revised the manuscript for intellectual content. MGH: performed statistical analyses or interpreted data, and revised the manuscript for intellectual content. EAT, DWD, JS, BO, and LP: revised the manuscript for intellectual content, contributed vital reagents/tools/patients, and obtained funding. RR and MVB: designed study, acquired data, analyzed or interpreted data, performed statistical analyses or interpreted data, drafted the manuscript for intellectual content, revised the manuscript for intellectual content, supervised or coordinated the study, and obtained funding. All authors read and approved the final manuscript.

\section{Funding}

The research leading to these results has received funding by the National Institutes of Health (NIH) grants R21 NS099631, R35 NS097261, R35 NS097273, P01 NS084974, P01 NS099114, as well as the ALS Association, Robert Packard Center for ALS Research, Muscular Dystrophy Association, and Mayo Clinic Foundation.

\section{Availability of data and materials}

All data relevant to the study are included in the article or as supplementary information. Upon reasonable request, additional information (e.g., protocols) will be shared by the corresponding authors.

Ethics approval and consent to participate

All subjects agreed to be in the study, and biological specimens were obtained after informed consent with approval from the Mayo Clinic Institutional Review Board (IRB).

\section{Competing interests}

MDJ and RR hold a patent on methods to screen for the hexanucleotide repeat expansion in the C9orf72 gene. No other competing interests were declared.

\section{Author details}

${ }^{1}$ Department of Neuroscience, Mayo Clinic, 4500 San Pablo Road, Jacksonville, FL 32224, USA. ²Department of Cancer Biology, Mayo Clinic, 4500 San Pablo Road, Jacksonville, FL 32224, USA. ${ }^{3}$ Division of Biomedical Statistics and Informatics, Mayo Clinic, 4500 San Pablo Road, Jacksonville, FL 32224, USA. ${ }^{4}$ Department of Neurology, Mayo Clinic, 4500 San Pablo Road, Jacksonville, FL 32224, USA. 
Received: 29 October 2019 Accepted: 14 January 2020

Published online: 30 January 2020

\section{References}

1. DeJesus-Hernandez M, Mackenzie IR, Boeve BF, Boxer AL, Baker M,

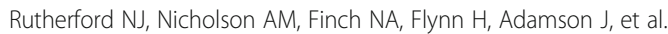
Expanded GGGGCC hexanucleotide repeat in noncoding region of C9ORF72 causes chromosome 9p-linked FTD and ALS. Neuron. 2011;72:24556.

2. Renton $A E$, Majounie E, Waite A, Simon-Sanchez J, Rollinson S, Gibbs JR, Schymick JC, Laaksovirta H, van Swieten JC, Myllykangas L, et al. A hexanucleotide repeat expansion in C9ORF72 is the cause of chromosome 9p21-linked ALS-FTD. Neuron. 2011;72:257-68.

3. Gendron TF, Bieniek KF, Zhang YJ, Jansen-West K, Ash PE, Caulfield T, Daughrity L, Dunmore JH, Castanedes-Casey M, Chew J, et al. Antisense transcripts of the expanded C9ORF72 hexanucleotide repeat form nuclear RNA foci and undergo repeat-associated non-ATG translation in C9FTD/ALS. Acta Neuropathol. 2013;126:829-44

4. Ash PE, Bieniek KF, Gendron TF, Caulfield T, Lin WL, Dejesus-Hernandez M, van Blitterswijk MM, Jansen-West K, Paul JW 3rd, Rademakers $R$, et al. Unconventional translation of C9ORF72 GGGGCC expansion generates insoluble polypeptides specific to C9FTD/ALS. Neuron. 2013;77:639-46.

5. Mori K, Weng SM, Arzberger T, May S, Rentzsch K, Kremmer E, Schmid B, Kretzschmar HA, Cruts M, Van Broeckhoven C, et al. The C9orf72 GGGGCC repeat is translated into aggregating dipeptide-repeat proteins in FTLD/ALS. Science. 2013:339:1335-8.

6. Mori K, Arzberger T, Grasser FA, Gijselinck I, May S, Rentzsch K, Weng SM, Schludi MH, van der Zee J, Cruts $\mathrm{M}$, et al. Bidirectional transcripts of the expanded C9orf72 hexanucleotide repeat are translated into aggregating dipeptide repeat proteins. Acta Neuropathol. 2013;126:881-93.

7. Zu T, Liu Y, Banez-Coronel M, Reid T, Pletnikova O, Lewis J, Miller TM, Harms $M B$, Falchook AE, Subramony SH, et al. RAN proteins and RNA foci from antisense transcripts in C9ORF72 ALS and frontotemporal dementia. Proc Natl Acad Sci U S A. 2013;110:E4968-77.

8. Xi Z, Zinman L, Moreno D, Schymick J, Liang Y, Sato C, Zheng Y, Ghani M, Dib S, Keith J, et al. Hypermethylation of the CpG island near the G4C2 repeat in ALS with a C9orf72 expansion. Am J Hum Genet. 2013:92:981-9.

9. Xi Z, Rainero I, Rubino E, Pinessi L, Bruni AC, Maletta RG, Nacmias B, Sorbi S, Galimberti D, Surace El, et al. Hypermethylation of the CpG-island near the C9orf72 G(4)C(2)-repeat expansion in FTLD patients. Hum Mol Genet. 2014; 23:5630-7.

10. Liu EY, Russ J, Wu K, Neal D, Suh E, McNally AG, Irwin DJ, Van Deerlin VM, Lee EB. C9orf72 hypermethylation protects against repeat expansionassociated pathology in ALS/FTD. Acta Neuropathol. 2014;128:525-41.

11. Russ J, Liu EY, Wu K, Neal D, Suh E, Irwin DJ, McMillan CT, Harms MB, Cairns $\mathrm{NJ}$, Wood EM, et al. Hypermethylation of repeat expanded C9orf72 is a clinical and molecular disease modifier. Acta Neuropathol. 2015;129:39-52.

12. Fournier C, Barbier M, Camuzat A, Anquetil V, Lattante S, Clot F, Cazeneuve C, Rinaldi D, Couratier P, Deramecourt V, et al. Relations between C9orf72 expansion size in blood, age at onset, age at collection and transmission across generations in patients and presymptomatic carriers. Neurobiol Aging. 2019:74:234 e231-8.

13. Beck J, Poulter M, Hensman D, Rohrer JD, Mahoney CJ, Adamson G, Campbell T, Uphill J, Borg A, Fratta P, et al. Large C9orf72 hexanucleotide repeat expansions are seen in multiple neurodegenerative syndromes and are more frequent than expected in the UK population. Am J Hum Genet. 2013;92:345-53.

14. van Blitterswijk M, Dejesus-Hernandez M, Niemantsverdriet $E$, Murray ME, Heckman MG, Diehl NN, Brown PH, Baker MC, Finch NA, Bauer PO, et al. Association between repeat sizes and clinical and pathological characteristics in carriers of C9ORF72 repeat expansions (Xpansize-72): a cross-sectional cohort study. Lancet Neurol. 2013;12:978-88.

15. Dols-lcardo O, Garcia-Redondo A, Rojas-Garcia R, Sanchez-Valle R, Noguera A, Gomez-Tortosa E, Pastor P, Hernandez I, Esteban-Perez J, Suarez-Calvet M, et al. Characterization of the repeat expansion size in C9orf72 in amyotrophic lateral sclerosis and frontotemporal dementia. Hum Mol Genet. 2014;23:749-54

16. Nordin A, Akimoto C, Wuolikainen A, Alstermark H, Jonsson P, Birve A, Marklund SL, Graffmo KS, Forsberg K, Brannstrom T, Andersen PM. Extensive size variability of the GGGGCC expansion in C9orf72 in both neuronal and non-neuronal tissues in 18 patients with ALS or FTD. Hum Mol Genet. 2015; 24:3133-42.

17. Suh E, Lee EB, Neal D, Wood EM, Toledo JB, Rennert L, Irwin DJ, McMillan $C T$, Krock B, Elman LB, et al. Semi-automated quantification of C9orf72 expansion size reveals inverse correlation between hexanucleotide repeat number and disease duration in frontotemporal degeneration. Acta Neuropathol. 2015;130:363-72.

18. Gijselinck I, Van Mossevelde S, van der Zee J, Sieben A, Engelborghs S, De Bleecker J, Ivanoiu A, Deryck O, Edbauer D, Zhang M, et al. The C9orf72 repeat size correlates with onset age of disease, DNA methylation and transcriptional downregulation of the promoter. Mol Psychiatry. 2016;21: $1112-24$.

19. van Blitterswijk M, Gendron TF, Baker MC, DeJesus-Hernandez M, Finch NA, Brown PH, Daughrity LM, Murray ME, Heckman MG, Jiang J, et al. Novel clinical associations with specific C9ORF72 transcripts in patients with repeat expansions in C9ORF72. Acta Neuropathol. 2015;130:863-76.

20. Haeusler AR, Donnelly CJ, Periz G, Simko EA, Shaw PG, Kim MS, Maragakis NJ, Troncoso JC, Pandey A, Sattler R, et al. C9orf72 nucleotide repeat structures initiate molecular cascades of disease. Nature. 2014;507:195-200.

21. Rizzu P, Blauwendraat C, Heetveld S, Lynes EM, Castillo-Lizardo M, Dhingra A, Pyz E, Hobert M, Synofzik M, Simon-Sanchez J, et al. C9orf72 is differentially expressed in the central nervous system and myeloid cells and consistently reduced in C9orf72, MAPT and GRN mutation carriers. Acta Neuropathol Commun. 2016:4:37.

22. McGoldrick $P$, Zhang M, van Blitterswijk M, Sato C, Moreno D, Xiao S, Zhang AB, McKeever PM, Weichert A, Schneider R, et al. Unaffected mosaic C9orf72 case: RNA foci, dipeptide proteins, but upregulated C9orf72 expression. Neurology. 2018;90:e323-31.

23. Xi Z, Zhang M, Bruni AC, Maletta RG, Colao R, Fratta P, Polke JM, Sweeney MG, Mudanohwo E, Nacmias B, et al. The C9orf72 repeat expansion itself is methylated in ALS and FTLD patients. Acta Neuropathol. 2015;129:715-27.

24. Cali CP, Patino M, Tai YK, Ho WY, McLean CA, Morris CM, Seeley WW, Miller BL, Gaig C, Vonsattel JPG, et al. C9orf72 intermediate repeats are associated with corticobasal degeneration, increased C9orf72 expression and disruption of autophagy. Acta Neuropathol. 2019:138:795-811.

25. Fratta P, Polke JM, Newcombe J, Mizielinska S, Lashley T, Poulter M, Beck J, Preza E, Devoy A, Sidle K, et al. Screening a UK amyotrophic lateral sclerosis cohort provides evidence of multiple origins of the C9orf72 expansion. Neurobiol Aging. 2015;36:546 e541-7.

26. Harper PS, Harley HG, Reardon W, Shaw DJ. Anticipation in myotonic dystrophy: new light on an old problem. Am J Hum Genet. 1992;51:10-6.

27. Van Mossevelde S, van der Zee J, Gijselinck I, Sleegers K, De Bleecker J, Sieben A, Vandenberghe R, Van Langenhove T, Baets J, Deryck O, et al. Clinical evidence of disease anticipation in families segregating a C9orf72 repeat expansion. JAMA Neurol. 2017;74:445-52.

28. Monros E, Molto MD, Martinez F, Canizares J, Blanca J, Vilchez JJ, Prieto F, de Frutos R, Palau F. Phenotype correlation and intergenerational dynamics of the Friedreich ataxia GAA trinucleotide repeat. Am J Hum Genet. 1997;61: $101-10$.

\section{Publisher's Note}

Springer Nature remains neutral with regard to jurisdictional claims in published maps and institutional affiliations.

Ready to submit your research? Choose BMC and benefit from:

- fast, convenient online submission

- thorough peer review by experienced researchers in your field

- rapid publication on acceptance

- support for research data, including large and complex data types

- gold Open Access which fosters wider collaboration and increased citations

- maximum visibility for your research: over $100 \mathrm{M}$ website views per year

At $\mathrm{BMC}$, research is always in progress.

Learn more biomedcentral.com/submission 